\title{
CONTACT DISTRIBUTION IN A THINNED BOOLEAN MODEL WITH POWER LAW RADII
}

\author{
YINGHUA DONG AND GENNADY SAMORODNITSKY
}

\begin{abstract}
We consider a weighted stationary spherical Boolean model in $\mathbb{R}^{d}$. Assuming that the radii of the balls in the Boolean model have regularly varying tails, we establish the asymptotic behaviour of the tail of the contact distribution of the thinned germ-grain model under 4 different thinning procedures of the original model.
\end{abstract}

\section{INTRODUCTION}

We consider hard-core global thinning of a stationary spherical Boolean model in $\mathbb{R}^{d}$, constructed as follows. Let $\Phi$ be a Poisson random measure on $\mathbb{R}^{d} \times(0, \infty) \times(0, \infty)$ with mean measure

$$
m(d \mathbf{x}, d r, d w)=\lambda d \mathbf{x} G(d r, d w) .
$$

Here $\lambda>0$ is the spatial intensity, and $G$ is a probability law on $(0, \infty) \times(0, \infty)$. Let $\left(\mathbf{X}_{n}, R_{n}, W_{n}\right)$, $n=1,2 \ldots$ be a measurable enumeration of the points of $\Phi$. We view $\mathbf{X}_{n} \in \mathbb{R}^{d}$ as the center of the $n$th ball, and $R_{n}$ its radius. In the sequel we will use the notation $B_{r}(\mathbf{x})$ for a closed ball of radius $r>0$ centered at $\mathbf{x} \in \mathbb{R}^{d}$, so the $n$th point of $\Phi$ corresponds to closed ball $B_{R_{n}}\left(\mathbf{X}_{n}\right)$. The last component, $W_{n}$, is the weight of the $n$th ball, and it will be used below in resolving collisions between balls. Let $F(\cdot)=G(\cdot \times(0, \infty))$ be the law of the radius marking a spatial Poissonian point. We will assume that

$$
\int_{0}^{\infty} r^{d} F(d r)<\infty
$$

It is well known that, under this assumption, with probability 1 , a realization of the random field $\Phi$ has the property that only finitely many balls of the type $B_{R_{n}}\left(\mathbf{X}_{n}\right)$ intersect any compact set in $\mathbb{R}^{d}$. This implies that the union

$$
D=\bigcup_{n=1}^{\infty} B_{R_{n}}\left(\mathbf{X}_{n}\right)
$$

is a random closed subset of $\mathbb{R}^{d}$. We refer the reader to Stoyan et al. (1995) for this fact, and for a general reference on Boolean and related models.

It is common to refer to a random configuration of the type we have constructed as a germ-grain model; such a model does not need to involve a spatial Poisson random measure or spherical shapes.

1991 Mathematics Subject Classification. Primary 60D05 .

Key words and phrases. Boolean model, hard-core thinning, power tails, contact distribution, empty space .

Dong's research was partially supported by Jiangsu Planned Projects for Postdoctoral Research Funds (No. 1501053A), the Statistical Scientific Research Program of National Bureau of Statistics of China (No. 2015LY83) and the foundation of Nanjing University of Information Science and Technology (No. 2014x026). Samorodnitsky's research was partially supported by the ARO grant W911NF-12-10385 and by the NSF grant DMS-1506783 at Cornell University. 
In the Boolean model above each $X_{n}$ is a germ, and the corresponding closed ball $B_{R_{n}}(\mathbf{0})$ is its grain. The set $D$ in (1.3) is the grain cover of the space.

Some of the balls $B_{R_{n}}\left(\mathbf{X}_{n}\right)$ in the Boolean model will overlap. In ordet to obtain a hard-core germ-grain model, i.e. a configuration in which no two grains overlap, it is possible to thin the Boolean model, by removing (at least) one ball in each pair of balls involved in an overlap. We will follow the global thinning procedure introduced by Mansson and Rudemo (2002). This is where the weight component $W_{n}$ of the $n$th ball is used. Informally, for every pair of different balls, $B_{R_{n}}\left(\mathbf{X}_{n}\right)$ and $B_{R_{m}}\left(\mathbf{X}_{m}\right)$ with a non-empty intersection, the ball $B_{R_{n}}\left(\mathbf{X}_{n}\right)$ gets deleted if $W_{n} \leq W_{m}$; this procedure deletes both balls if $W_{n}=W_{m}$. To be a bit more formal, we use the notation borrowed from Kuronen and Leskelä (2013): let

$$
N_{\mathbf{x}, r, w}=\left\{\left(\mathbf{x}^{\prime}, r^{\prime}, w^{\prime}\right) \in \mathbb{R}^{d} \times \mathbb{R}_{+} \times \mathbb{R}_{+} \backslash\{\mathbf{x}, r, w\}: B_{r^{\prime}}\left(\mathbf{x}^{\prime}\right) \cap B_{r}(x) \neq \emptyset\right\} ;
$$

we view this set as the collection of centers, radii and weights of balls that could, potentially, intersect a reference ball $B_{r}(\mathbf{x})$ with weight $w$. Then the thinned Boolean model we are considering is given by

$$
\Phi^{\text {th }}=\left\{(\mathbf{x}, r, w) \in \Phi: w>w^{\prime}, \text { for all }\left(\mathbf{x}^{\prime}, r^{\prime}, w^{\prime}\right) \in \Phi \cap N_{\mathbf{x}, r, w}\right\} .
$$

By construction, all the remaining grains (balls) in the thinned random field $\Phi^{\text {th }}$ are disjoint. The corresponding grain cover can be written in the form

$$
D_{\mathrm{th}}=\bigcup_{(\mathbf{x}, r, w) \in \Phi^{\mathrm{th}}} B_{r}(\mathbf{x}) .
$$

The thinning procedure we are using is sometimes referred to as the Matérn type II construction. An discussion of different Matérn type constructions and their extensions was given in Nguyen and Baccelli (2013). This model was studied in Mansson and Rudemo (2002) and Anderson et al. (2006). Our inspiration for the present work came from the paper of Kuronen and Leskelä (2013), and we refer the reader to this paper for an illuminating discussion of the importance and applications of hard-core germ-grain models. Specifically, the latter paper considers the case of power law grain sizes; in our notation we can describe this setup as follows. Recalling that we denote by $F$ the marginal distribution of the probability measure $G$ in (1.1) corresponding to the random radius of a Poisson ball, the power law distribution of the grain sizes in Kuronen and Leskelä (2013) is the assumption of regular variation of the tail

$$
\bar{F}(r):=1-F(r)=r^{-\alpha} L(r),
$$

where $L$ is a slowly varying function and $\alpha>d$; the latter restriction assures that the integrability condition (1.2) holds. We refer the reader to Resnick (2007) for information on regular varying tails. Under the assumption (1.7) of regular variation, Kuronen and Leskelä (2013) discovered appearance of power-like decay of the covariance function of the thinned grain cover (1.6), under three out of four choices of the joint law $G$ in (1.1) they considered; we will return to these choices in a moment.

In this paper we are interested in the contact distribution for the thinned Boolean model described above. It is a probability law $H$ on $(0, \infty)$ whose complementary c.d.f. is defined by

$$
\bar{H}(r)=P\left(B_{r}(\mathbf{0}) \cap D_{\text {th }}=\emptyset \mid \mathbf{0} \notin D_{\text {th }}\right), r>0 .
$$

The contact distribution can, of course, be defined for any germ-grain model. It differs only by a possible atom at zero from the empty space function, a probability law on $[0, \infty)$ defined by

$$
\bar{H}_{\mathrm{e}}(r)=P\left(B_{r}(\mathbf{0}) \cap D_{\mathrm{th}}=\emptyset\right), r \geq 0 .
$$


Contact distributions are important characteristics of germ-grain models; a survey on the topic is in Hug et al. (2002). Explicit formulas for the contact distributions are mostly available only for Poisson-based models such as Poisson cluster models. Our goal in this paper is to understand the tail behaviour of the contact distribution for the thinned Boolean model with a power law distribution of the grain sizes. Specifically, we are interested in answering the question whether a power law distribution of the grain sizes results in a power law behaviour of the contact distribution for the thinned Boolean model. Notice that for the original Boolean model with the grain cover (1.3) the tail of the contact distribution decays, obviously, exponentially fast regardless of the distribution of the radius of a ball. It turns out that certain choices of the joint law $G$ of the radius of a ball and its weight lead to appearance of a power law-like decay of the contact distribution, while other choices do not.

One possible choice of the the law $G$ in (1.1) is given by setting $W_{n}=R_{n}$ a.s. for all $n$, so that $G$ is concentrated on the diagonal $r=w$ of $(0, \infty) \times(0, \infty)$. With this choice of $G$, balls with a larger radius have a larger weight. We refer to this situation as the case of heavy large balls. Another possible choice of $G$ is given by setting $W_{n}=1 / R_{n}$ a.s. for all $n$. With this choice of $G$, balls with a smaller radius have a larger weight. A third possible choice of the law $G$ is to make it a product law, and to make the marginal law of the weights continuous (e.g. standard uniform). That is, the weights are independent of the radii of the balls. Finally, one could make the weights of the balls constant (e.g. $W_{n}=1$ a.s. for all $n$ ). In this case, only isolated balls in the original Boolean model (i.e. the balls that do not overlap with any other ball) stay in the thinned grain-germ model $\Phi^{\text {th }}$. The latter thinning mechanism is known as the Matérn type I construction.

It is interesting that, as it was shown in Kuronen and Leskelä (2013), when the radii of the balls are regularly varying as in (1.7), the covariance function of the thinned grain cover (1.6) has a power-like decay under all of the above thinning mechanisms apart from the case of heavy small balls.

In a certain sense the above situation is preserved when one is interested in the tail of the contact distribution. In Section 2 we show that this tail has a power-like decay in all cases apart from the case of heavy small balls. Interestingly, the power of the rate of decay is different for the three thinning mechanisms considered here. Finally, In Section 3, on the other hand, we show that in the situation when a ball with a large radius has a smaller weight than a ball with a small radius, the tail of the contact distribution decays exponentially fast.

\section{Power law of the CONTACT Distribution}

We start this section with showing that, in the case when a ball with a larger radius in a Boolean model has a larger weight than a ball with a smaller radius, the contact distribution of the thinned model has a tail with power-like decay.

Theorem 2.1. Assume that the distribution of the radii of the balls in the Boolean model satisfies (1.7) with $\alpha>d$. If larger balls have larger weights, then the contact distribution of the thinned germ-grain model satisfies

$$
0<\liminf _{r \rightarrow \infty} \frac{\bar{H}(r)}{\left(r^{d} \bar{F}(r)\right)^{2}} \leq \limsup _{r \rightarrow \infty} \frac{\bar{H}(r)}{\left(r^{d} \bar{F}(r)\right)^{2}}<\infty .
$$

Proof. Throughout the proof, we may and will work with the tail of the empty space function (1.9) instead of the tail of the contact distribution. Further, we denote by $c$ a finite positive constant whose value is not important and that may change from one appearance to the next. We will also introduce a notational simplification. The Poisson random measure $\Phi$ is a measure in the $d+2$ dimensional space $\mathbb{R}^{d} \times(0, \infty) \times(0, \infty)$, but in the present context the "weight" coordinate is a 
function of the "radius" coordinate, so it is simpler to view $\Phi$ as a measure in the $d+1$-dimensional space $\mathbb{R}^{d} \times(0, \infty)$, described by the location of the center of a ball and its radius. We will use the appropriate notation throughout the proof.

We start with proving the lower bound in (2.1). We will construct a scenario under which the ball $B_{r}(\mathbf{0})$ does not intersect $\Phi^{\text {th }}$. The idea of the construction is that a single ball with a large radius in $\Phi$ "eliminates" all the other balls in $\Phi$ that intersect $B_{r}(\mathbf{0})$, and then another ball in $\Phi$ of an even larger radius "eliminates" the first ball of a large radius, but does not itself intersect $B_{r}(\mathbf{0})$. That will leave $B_{r}(\mathbf{0})$ disjoint from $\Phi^{\text {th }}$. The two large balls will have centers in sets of sizes proportional to $r$, and also radii of the size proportional to $r$, which explains the order of magnitude of the tail in (2.1).

For $r>0$ we consider three disjoint subsets of $\mathbb{R}^{d} \times(0, \infty)$ :

$$
\begin{gathered}
A_{r}^{(1)}=\{(\mathbf{x}, t): t \geq\|\mathbf{x}\|+r\}, \\
A_{r}^{(2)}=\{(\mathbf{x}, t): \max (r,\|\mathbf{x}\|-r) \leq t<\|\mathbf{x}\|+r\}, \\
A_{r}^{(3)}=\{(\mathbf{x}, t):\|\mathbf{x}\|-r \leq t<r\} .
\end{gathered}
$$

Notice that only those balls $B_{R_{n}}\left(\mathbf{X}_{n}\right)$ in $\Phi$ for which $\left(X_{n}, R_{n}\right) \in A_{r}^{(1)} \cup A_{r}^{(2)} \cup A_{r}^{(3)}$ intersect $B_{r}(\mathbf{0})$. Furthermore, any ball $B_{R_{n}}\left(\mathbf{X}_{n}\right)$ in $\Phi$ for which $\left(X_{n}, R_{n}\right) \in A_{r}^{(1)}$ contains the entire ball $B_{r}(\mathbf{0})$ as a subset. The set $A_{r}^{(1)}$ will be most important for us in proving the lower bound in (2.1). Consider the event

$$
B_{r}=\left\{\Phi\left(A_{r}^{(1)}\right)=1, \Phi\left(A_{r}^{(2)}\right)=0\right\} .
$$

On the event $B_{r}$ we can define a random vector $\left(\mathbf{X}^{(r)}, R^{(r)}\right) \in A_{r}^{(1)}$ corresponding to the location of the center and the radius of the single ball in $\Phi$ for which that pair is in the set $A_{r}^{(1)}$. We extend the definition of $\left(\mathbf{X}^{(r)}, R^{(r)}\right)$ to the outside of the event $B_{r}$ in an arbitrary measurable way (e.g. define it on $B_{r}^{c}$ to be the pair $(\mathbf{0}, 1)$.) Clearly, this vector has the law

$$
P\left(\left(\mathbf{X}^{(r)}, R^{(r)}\right) \in \cdot \mid B_{r}\right)=\frac{(\operatorname{Leb} \times F)(\cdot)}{(\operatorname{Leb} \times F)\left(A_{r}^{(1)}\right)}
$$

over $A_{r}^{(1)}$. Here Leb is the $d$-dimensional Lebesgue measure. Note that

$$
\bar{H}_{\mathrm{e}}(r)=P\left(B_{r}(\mathbf{0}) \cap D_{\mathrm{th}}=\emptyset\right) \geq P\left(B_{r} \cap \hat{B}_{r}\right),
$$

where

$$
\begin{aligned}
\hat{B}_{r}=\left\{\text { there is a } \Phi \text {-ball } B_{R_{n}}\left(\mathbf{X}_{n}\right) \text { with }\left(\mathbf{X}_{n}, R_{n}\right) \in\left(A_{r}^{(1)} \cup A_{r}^{(2)} \cup A_{r}^{(3)}\right)^{c}\right. \\
\\
\text { and } \left.R_{n}>R^{(r)} \text { that intersects } B_{R^{(r)}}\left(\mathbf{X}^{(r)}\right) .\right\}
\end{aligned}
$$

Let $v_{d}$ be the volume of the unit ball in $\mathbb{R}^{d}$. By switching to the spherical coordinates we see that for the large $r$,

$$
\begin{aligned}
E\left[\Phi\left(A_{r}^{(1)}\right)\right] & =\lambda d v_{d} \int_{0}^{\infty} x^{d-1} \bar{F}(x+r) d x \\
& =\lambda d v_{d} r^{d} \int_{0}^{\infty} t^{d-1} \bar{F}(r(t+1)) d t \\
& \sim c r^{d} \bar{F}(r) \rightarrow 0 .
\end{aligned}
$$


On the last step we used the Potter bounds for regularly varying functions; see Resnick (2007). Therefore, for large $r$,

$$
P\left(B_{r}\right) \sim E\left[\Phi\left(A_{r}^{(1)}\right)\right] \sim c r^{d} \bar{F}(r) .
$$

Similarly,

$$
E\left[\Phi\left(A_{r}^{(2)}\right)\right] \sim c r^{d} \bar{F}(r) \rightarrow 0
$$

as $r \rightarrow \infty$.

Next, for $(\mathbf{y}, w) \in A_{r}^{(1)}$ we denote

$$
A_{r,(\mathbf{y}, w)}=\left\{(\mathbf{x}, t) \in\left(A_{r}^{(1)} \cup A_{r}^{(2)} \cup A_{r}^{(3)}\right)^{c}: t>w \text {, the ball } B_{t}(\mathbf{x}) \text { intersects the ball } B_{w}(\mathbf{y})\right\} \text {. }
$$

Then, since a Poisson random measure assigns independent values to disjoint sets,

$$
\begin{aligned}
P\left(\hat{B}_{r} \mid B_{r}\right) & =\frac{1}{(\operatorname{Leb} \times F)\left(A_{r}^{(1)}\right)} \iint_{(\mathbf{y}, w) \in A_{r}^{(1)}} P\left(\Phi\left(A_{r,(\mathbf{y}, w)}>0\right) d \mathbf{y} F(d w)\right. \\
& \geq \frac{1}{(\operatorname{Leb} \times F)\left(A_{r}^{(1)}\right)} \int_{B_{3 r}(\mathbf{0}) \backslash B_{2 r}(\mathbf{0})} \int_{5 r}^{5.5 r}\left(1-\exp \left\{-m\left(A_{r,(\mathbf{y}, w)}\right)\right\}\right) d \mathbf{y} F(d w)
\end{aligned}
$$

because, obviously,

It follows from (2.6) that

$$
\left(B_{3 r}(\mathbf{0}) \backslash B_{2 r}(\mathbf{0})\right) \times(5 r, 5.5 r) \subset A_{r}^{(1)} .
$$

$$
\frac{(\operatorname{Leb} \times F)\left[\left(B_{3 r}(\mathbf{0}) \backslash B_{2 r}(\mathbf{0})\right) \times(5 r, 5.5 r)\right]}{(\operatorname{Leb} \times F)\left(A_{r}^{(1)}\right)} \geq c
$$

for all large $r$. Therefore, the lower bound in (2.1) will follow from (2.5) and (2.7) once we show that there is a constant $c$ such that for all $r$ large enough

$$
m\left(A_{r,(\mathbf{y}, w)}\right) \geq c r^{d} \bar{F}(r)
$$

for all $(\mathbf{y}, w) \in\left(B_{3 r}(\mathbf{0}) \backslash B_{2 r}(\mathbf{0})\right) \times(5 r, 5.5 r)$. To this end, for such a pair $(\mathbf{y}, w)$ consider the point

$$
\tilde{\mathbf{y}}=\frac{\mathbf{y}}{\|\mathbf{y}\|}(\|\mathbf{y}\|+w) \in \mathbb{R}^{d},
$$

and the ball $B_{r}(\tilde{\mathbf{y}})$. Let $\|\mathbf{z}\| \leq r$. Note that the distance from the point $\tilde{\mathbf{y}}+\mathbf{z} \in B_{r}(\tilde{\mathbf{y}})$ to the ball $B_{w}(\mathbf{y})$ does not exceed $\|\mathbf{z}\| \leq r$, while the distance from that same point to ball $B_{r}(\mathbf{0})$ is greater than $\|\mathbf{y}\|+w-r>w+r$. Taking into account the bounds on $w$ we have chosen, we see that any ball centered at a point $\tilde{\mathbf{y}}+\mathbf{z} \in B_{r}(\tilde{\mathbf{y}})$ with a radius $t \in(5.5 r, 6 r)$ will intersect the ball $B_{w}(\mathbf{y})$ but not the ball $B_{r}(\mathbf{0})$. We conclude that for a pair $(\mathbf{y}, w)$ as above,

$$
A_{r,(\mathbf{y}, w)} \supset\left\{(\mathbf{x}, t): \mathbf{x} \in B_{r}(\tilde{\mathbf{y}}), t \in(5.5 r, 6 r)\right\},
$$

implying that

$$
m\left(A_{r,(\mathbf{y}, w)}\right) \geq c r^{d}(\bar{F}(5.5 r)-\bar{F}(6 r)) \sim c r^{d} \bar{F}(r)
$$

as $r \rightarrow \infty$, by the regular variation. This proves (2.8).

Now we switch to proving the upper bound in (2.1). Let $K>0$ be a fixed number to be specified momentarily. Denote

$$
A_{r}^{(4)}(K)=\{(\mathbf{x}, t): \max (r / K,\|\mathbf{x}\|-r) \leq t<\|\mathbf{x}\|+r\} .
$$

The same argument using regular variation and the Potter bounds as in (2.6) shows that for large $r$,

$$
E\left[\Phi\left(A_{r}^{(4)}(K)\right)\right] \leq c r^{d} \bar{F}(r)
$$


(with a $K$-dependent constant $c$ ). This bound, together with (2.6), tells us that for large $r$,

$$
\begin{gathered}
P\left(\Phi\left(A_{r}^{(1)}\right) \geq 2\right) \leq c\left(r^{d} \bar{F}(r)\right)^{2}, \quad P\left(\Phi\left(A_{r}^{(4)}(K)\right) \geq 2\right) \leq c\left(r^{d} \bar{F}(r)\right)^{2}, \\
P\left(\Phi\left(A_{r}^{(1)}\right) \geq 1, \Phi\left(A_{r}^{(4)}(K)\right) \geq 1\right) \leq c\left(r^{d} \bar{F}(r)\right)^{2} .
\end{gathered}
$$

Therefore, the upper bound in (2.1) will follow once we prove the following 3 statements. For large $r$,

$$
\begin{gathered}
P\left(B_{r}(\mathbf{0}) \cap D_{\text {th }}=\emptyset, \Phi\left(A_{r}^{(1)}\right)=1\right) \leq c\left(r^{d} \bar{F}(r)\right)^{2}, \\
P\left(B_{r}(\mathbf{0}) \cap D_{\text {th }}=\emptyset, \Phi\left(A_{r}^{(4)}(K)\right)=1\right) \leq c\left(r^{d} \bar{F}(r)\right)^{2}, \\
P\left(B_{r}(\mathbf{0}) \cap D_{\text {th }}=\emptyset, \Phi\left(A_{r}^{(1)}\right)=\Phi\left(A_{r}^{(4)}(K)\right)=0\right) \leq c\left(r^{d} \bar{F}(r)\right)^{2} .
\end{gathered}
$$

We will see that (2.10) and (2.11) hold for any $K>0$. We will specify $K$ when we prove (2.12).

We start with proving (2.10). For the event in that probability to occur, the only $\Phi$-ball in $A_{r}^{(1)}$ must overlap with another $\Phi$-ball, of a larger radius, and lying outside of $A_{r}^{(1)}$. Since restrictions of a Poisson measure to disjoint sets are independent, and since the only $\Phi$-ball in $A_{r}^{(1)}$ has a radius of at least $r$, the probability in (2.10) is bounded from above by

$$
P\left(\Phi\left(A_{r}^{(1)}\right)=1\right) \sup _{s \geq r} P\left(\Phi\left(A_{s}^{(5)}\right)>0\right),
$$

where

$$
A_{s}^{(5)}=\left\{(\mathbf{x}, t) \in \mathbb{R}^{d} \times(0, \infty): t>s, \text { the ball } B_{t}(\mathbf{x}) \text { intersects the ball } B_{s}(\mathbf{0})\right\},
$$

the center of the ball of radius $s$ being irrelevant due to the stationarity. It is elementary that for large $s$, by the regular variation of $\bar{F}$ and Karamata's theorem on integration of regularly varying functions (see e.g. Resnick (2007)),

$$
\begin{aligned}
m\left(A_{s}^{(5)}\right) & =c \int_{0}^{\infty} x^{d-1} \bar{F}(s \vee(x-s)) d x \\
& =c(2 s)^{d} \bar{F}(s)+c \int_{2 s}^{\infty} x^{d-1} \bar{F}(x-s) d x \\
& \leq c s^{d} \bar{F}(s) .
\end{aligned}
$$

Therefore, for large $s$,

$$
P\left(\Phi\left(A_{s}^{(5)}\right)>0\right) \leq c s^{d} \bar{F}(s),
$$

and (2.10) follows from (2.6). Clearly, all the ingredients involved in the proof of (2.10) are also available for the proof of $(2.11)$, so we only need to prove (2.12).

Now we explain how to choose $K$. It will be chosen together with several other constants. Choose sequentially positive real numbers $0<\theta<d / \alpha$ and $0<\tau<\theta(\alpha-d)$, a positive integer $I>2(\alpha-d) / \tau-1$ and, finally, $K>2^{I+1}$. We start with considering the concentric balls $B_{r 2^{-i}}(\mathbf{0})$, $i=0,1, \ldots, I$. For $i=0,1, \ldots, I$, let

$$
M_{i}=\sup \left\{R_{n}: B_{R_{n}}\left(\mathbf{X}_{n}\right) \text { is an } \Phi \text {-ball, }\left\|\mathbf{X}_{n}\right\|+R_{n}<r 2^{-i}\right\} .
$$

Then

$$
P\left(M_{i} \leq\left(r 2^{-i}\right)^{\theta}\right)=\exp \left\{-m\left(\left\{(\mathbf{x}, t):\|\mathbf{x}\|+t<r 2^{-i}, t>\left(r 2^{-i}\right)^{\theta}\right\}\right)\right\}
$$


Further, since $\theta<1$,

$$
\begin{aligned}
& m\left(\left\{(\mathbf{x}, t):\|\mathbf{x}\|+t<r, t>r^{\theta}\right\}\right) \\
\geq & m\left(\left\{(\mathbf{x}, t):\|\mathbf{x}\|<r / 2, r^{\theta}<t \leq r / 2\right\}\right) \\
= & c r^{d}\left(\bar{F}\left(r^{\theta}\right)-\bar{F}(r / 2)\right) \sim c r^{d} \bar{F}\left(r^{\theta}\right)
\end{aligned}
$$

as $r \rightarrow \infty$. By the choice of $\theta$ we see that

$$
P\left(M_{i} \leq\left(r 2^{-i}\right)^{\theta}\right)=o\left(\left(r^{d} \bar{F}(r)\right)^{2}\right), i=0,1, \ldots, I,
$$

and so (2.12) will follow once we prove that

$$
\limsup _{r \rightarrow \infty} \frac{P\left(B_{r}(\mathbf{0}) \cap D_{\mathrm{th}}=\emptyset, \Phi\left(A_{r}^{(1)}\right)=\Phi\left(A_{r}^{(4)}(K)\right)=0, M_{i}>\left(r 2^{-i}\right)^{\theta}, i=0,1, \ldots, I\right)}{\left(r^{d} \bar{F}(r)\right)^{2}}<\infty .
$$

Consider the events

$$
\begin{aligned}
H_{i}= & \left\{\text { the } \Phi \text {-ball fully inside } B_{r 2^{-i}}(\mathbf{0})\right. \text { of the largest radius, } \\
& \text { is eliminated by an } \left.\Phi \text {-ball not fully inside } B_{r 2^{-i}}(\mathbf{0})\right\},
\end{aligned}
$$

$i=0,1, \ldots, I$. Note that, on the event $H_{i}^{c}$, the largest $\Phi$-ball fully inside $B_{r 2^{-i}}(\mathbf{0})$ stays in the thinned process, hence $B_{r}(\mathbf{0}) \cap D_{\text {th }} \neq \emptyset$. Therefore, in order to prove (2.15), it is enough to prove that

$$
\begin{gathered}
\limsup _{r \rightarrow \infty}\left(r^{d} \bar{F}(r)\right)^{-2} P\left(\left\{\Phi\left(A_{r}^{(1)}\right)=\Phi\left(A_{r}^{(4)}(K)\right)=0, M_{i}>\left(r 2^{-i}\right)^{\theta}, i=0,1, \ldots, I\right\}\right. \\
\left.\cap H_{0} \cap \ldots \cap H_{I}\right)<\infty .
\end{gathered}
$$

Consider first the probability

$$
\begin{aligned}
& P\left(\left\{\Phi\left(A_{r}^{(1)}\right)=\Phi\left(A_{r}^{(4)}(K)\right)=0, M_{i}>\left(r 2^{-i}\right)^{\theta}, i=0,1, \ldots, I\right\} \cap H_{I}\right) \\
\leq & P\left(\left\{\Phi\left(A_{r}^{(1)}\right)=\Phi\left(A_{r}^{(4)}(K)\right)=0, M_{I}>\left(r 2^{-I}\right)^{\theta}\right\} \cap H_{I}\right) .
\end{aligned}
$$

On the latter event, we can define a random vector $\left(\tilde{\mathbf{X}}_{I}, \tilde{R}_{I}\right)$ as the center and the radius of the largest $\Phi$-ball fully within $B_{r 2^{-I}}(\mathbf{0})$. Note that $\tilde{R}_{I}>\left(r 2^{-I}\right)^{\theta}$. The random vector $\left(\tilde{\mathbf{X}}_{I}, \tilde{R}_{I}\right)$ is determined by the field $\Phi$ on the set

$$
\left\{(\mathbf{x}, t):\|\mathbf{x}\|+t<r 2^{-I}\right\}
$$

and the corresponding $\Phi$-ball can only be eliminated by the $\Phi$-balls in the complement of that set. Since restrictions of a Poisson measure to disjoint sets are independent, we conclude, in the notation of (2.13) that for large $r$,

$$
\begin{aligned}
P\left(\left\{\Phi\left(A_{r}^{(1)}\right)=\Phi\left(A_{r}^{(4)}(K)\right)=0, M_{I}>\left(r 2^{-I}\right)^{\theta}\right\} \cap H_{I}\right) & \leq \sup _{s \geq\left(r 2^{-I}\right)^{\theta}} P\left(\Phi\left(A_{s}^{(5)}\right)>0\right) \\
& \leq c\left(r 2^{-I}\right)^{\theta d} \bar{F}\left(\left(r 2^{-I}\right)^{\theta}\right) \leq c r^{-\tau}
\end{aligned}
$$

where on the last two steps we used (2.14), the choice of $\tau$ and the regular variation of $\bar{F}$. 
Next we consider the probability

$$
\begin{aligned}
& \left.P\left(\Phi\left(A_{r}^{(1)}\right)=\Phi\left(A_{r}^{(4)}(K)\right)=0, M_{i}>\left(r 2^{-i}\right)^{\theta}, i=0,1, \ldots, I\right\} \cap H_{I-1} \cap H_{I}\right) \\
\leq & \left.P\left(\Phi\left(A_{r}^{(1)}\right)=\Phi\left(A_{r}^{(4)}(K)\right)=0, M_{i}>\left(r 2^{-i}\right)^{\theta}, i=I-1, I\right\} \cap H_{I-1} \cap H_{I}\right) .
\end{aligned}
$$

Note that the condition $\Phi\left(A_{r}^{(1)}\right)=\Phi\left(A_{r}^{(4)}(K)\right)=0$ in the above event means that the largest $\Phi$-ball completely within $B_{r 2^{-I}}(\mathbf{0})$ could only be eliminated by a $\Phi$-ball centered at a point whose norm is in the range $r 2^{-I} \pm r / K$, while the largest $\Phi$-ball completely within $B_{r 2^{-(I-1)}}(\mathbf{0})$ could only be eliminated by a $\Phi$-ball centered at a point whose norm is in the range $r 2^{-(I-1)} \pm r / K$. These two ranges are disjoint by the choice of $K$. We use, once again, the fact that restrictions of a Poisson measure to disjoint sets are independent, and an argument as above gives us

$$
\left.P\left(\Phi\left(A_{r}^{(1)}\right)=\Phi\left(A_{r}^{(4)}(K)\right)=0, M_{i}>\left(r 2^{-i}\right)^{\theta}, i=I-1, I\right\} \cap H_{I-1} \cap H_{I}\right) \leq c\left(r^{-\tau}\right)^{2} .
$$

Proceeding in the same manner, we finally obtain

$$
P\left(\left\{\Phi\left(A_{r}^{(1)}\right)=\Phi\left(A_{r}^{(4)}(K)\right)=0, M_{i}>\left(r 2^{-i}\right)^{\theta}, i=0,1, \ldots, I\right\} \cap H_{0} \cap \ldots \cap H_{I}\right) \leq c\left(r^{-\tau}\right)^{I+1}
$$

for large $r$. By the choice of $I$, we see that (2.16) follows.

Now we consider the case of isolated balls remaining. Once again, the contact distribution has a power-like decaying tail, but the corresponding power is different from the power obtained in Theorem 2.1. This is, perhaps, not surprising, since keeping only isolated balls results in fewest balls remaining in the thinned model, hence larger "open space".

Theorem 2.2. Assume that the distribution of the radii of the balls in the Boolean model satisfies (1.7) with $\alpha>d$. If only isolated balls are kept in the thinned model, then the contact distribution of the thinned germ-grain model satisfies

$$
0<\liminf _{r \rightarrow \infty} \frac{\bar{H}(r)}{r^{d} \bar{F}(r)} \leq \limsup _{r \rightarrow \infty} \frac{\bar{H}(r)}{r^{d} \bar{F}(r)}<\infty .
$$

Proof. We use the same conventions as in the proof of Theorem 2.1. In particular, we work with the tail of the empty space function, and view the Poisson random measure $\Phi$ as a measure in the $d+1$-dimensional space $\mathbb{R}^{d} \times(0, \infty)$.

Once again, we start with a lower bound. One scenario under which the ball $B_{r}(\mathbf{0})$ is disjoint from the grain cover in the thinned model is existence of a $\Phi$-ball that covers the entire ball $B_{r}(\mathbf{0})$ plus existence of a $\Phi$-ball that is entirely within the ball $B_{r}(\mathbf{0})$. Since

$$
m\left(\left\{(\mathbf{x}, t): B_{t}(\mathbf{x}) \subset B_{r}(\mathbf{0})\right\}\right) \rightarrow \infty
$$

as $r \rightarrow \infty$, we conclude by (2.6) that

$$
\begin{aligned}
& P\left(B_{r}(\mathbf{0}) \cap D_{\mathrm{th}}=\emptyset\right) \geq(1-o(1)) P\left(\Phi\left(A_{r}^{(1)}\right) \geq 1\right) \\
\sim & E\left(\Phi\left(A_{r}^{(1)}\right)\right) \sim c r^{d} \bar{F}(r) .
\end{aligned}
$$

This proves the lower bound in (2.17).

The argument for the upper bound in (2.17) is based on several facts. First of all, since the thinned random filed $\Phi^{\text {th }}$ is a.s. non-empty, for any $\varepsilon>0$ and large enough $a>0$,

$$
P\left(\text { there is } B_{v}(\mathbf{x}) \in \Phi^{\text {th }} \text { with }\|\mathbf{x}\| \leq \varepsilon \text { and } v \leq a\right)>0 .
$$


Secondly, there is a constant $c>0$ such that for any $0<a \leq r$ there exist at least $\left[\mathrm{cr}^{d} / a^{d}\right]$ closed balls of radius $a$ completely within $B_{r}(\mathbf{0})$, such that the Euclidian distance between any two different balls is at least $a$. This fact can be easily verified by considering a regular grid of size $a$ inside $B_{r}(\mathbf{0})$.

Let $M(r)$ be the largest radius of a $\Phi$-ball intersecting $B_{r}(\mathbf{0})$. Clearly, for any $t>0$,

$$
P(M(r)>t)=1-e^{-m\left(A_{r, t}^{(6)}\right)},
$$

where

$$
A_{r, t}^{(6)}=\left\{(\mathbf{x}, s) \in \mathbb{R}^{d} \times(0, \infty): s>t \text {, the ball } B_{s}(\mathbf{x}) \text { intersects the ball } B_{r}(\mathbf{0})\right\} .
$$

An argument similar to the one in (2.14) shows that

$$
m\left(A_{r, t}^{(6)}\right) \leq c r^{d} \bar{F}(t),
$$

with a similar lower bound, but with a different constant $c$. Write

$$
P\left(B_{r}(\mathbf{0}) \cap D_{\mathrm{th}}=\emptyset\right) \leq P(M(r)>r)+\int_{0}^{r} P\left(B_{r}(\mathbf{0}) \cap D_{\mathrm{th}}=\emptyset \mid M(r)=t\right) F_{M(r)}(d t),
$$

where $F_{M(r)}$ is the law of $M(r)$. It follows from (2.19) that we have to prove that

$$
\limsup _{r \rightarrow \infty} \frac{\int_{0}^{r} P\left(B_{r}(\mathbf{0}) \cap D_{\mathrm{th}}=\emptyset \mid M(r)=t\right) F_{M(r)}(d t)}{r^{d} \bar{F}(r)}<\infty .
$$

The property that restrictions of a Poisson measure to disjoint sets are independent tells us that, given that $M(r)=t$, the random measure $\Phi$ restricted to the set $A_{t}^{(6)}=\{(\mathbf{x}, s): s<t\}$ is still a Poisson random measure on that set with the same mean measure $m$, restricted to that set. Take $a>0$ such that (2.18) holds, and choose $\varepsilon=a$. Let $0<p<1$ be the corresponding value of the probability in (2.18). Consider $a<t<r$. By (2.18), there are $\left[c r^{d} / t^{d}\right]$ closed balls of radius $t$ completely within $B_{r}(\mathbf{0})$, such that the Euclidian distance between any two different balls is at least $t$. For each one of these $\left[c r^{d} / t^{d}\right]$ balls, with probability at least $p$, there is an isolated $\Phi$-ball with a center in it, and radius not exceeding $t$. The events that such $\Phi$-balls exist are independent, and presence of such a $\Phi$-ball guarantees that $B_{r}(\mathbf{0}) \cap D_{\text {th }} \neq \emptyset$. Therefore, for any $t>a$,

$$
P\left(B_{r}(\mathbf{0}) \cap D_{\text {th }}=\emptyset \mid M(r)=t\right) \leq(1-p)^{\left[c r^{d} / t^{d}\right]} \leq(1-p)^{-1}(1-p)^{c r^{d} / t^{d}} .
$$

It is clear that

$$
P(M(r) \leq a) \leq e^{-c r^{d}}=o\left(r^{d} \bar{F}(r)\right)
$$

Furthermore,

$$
\begin{aligned}
& \int_{a}^{r} P\left(B_{r}(\mathbf{0}) \cap D_{\mathrm{th}}=\emptyset \mid M(r)=t\right) F_{M(r)}(d t) \\
\leq & c \int_{a}^{r} e^{-r^{d} / c t^{d}} F_{M(r)}(d t) \\
\leq & c r^{d} \int_{0}^{r} e^{-r^{d} / c t^{d}} t^{-(d+1)} \bar{F}_{M(r)}(t) d t \\
\leq & c r^{2 d} \int_{0}^{r} e^{-r^{d} / c t^{d}} t^{-(d+1)} \bar{F}(t) d t \\
= & c r^{d} \int_{0}^{1} e^{-1 / c s^{d}} s^{-(d+1)} \bar{F}(r s) d s
\end{aligned}
$$




$$
\sim c r^{d} \bar{F}(r) \int_{0}^{1} e^{-1 / c s^{d}} s^{-(\alpha+d+1)} d s
$$

as $r \rightarrow \infty$ by the regular variation of $\bar{F}$ and the Potter bounds. This completes the proof of (2.20) and, hence, of the upper bound in the theorem.

Finally, we consider the case when the weights are independent of the radii of the balls.

Theorem 2.3. Assume that the distribution of the radii of the balls in the Boolean model satisfies (1.7) with $\alpha>d$. If the weight of a ball in the model is independent of its radius and has a continuous distribution, then the contact distribution of the thinned germ-grain model satisfies

$$
0<\liminf _{r \rightarrow \infty} \frac{\bar{H}(r)}{\bar{F}(r)} \leq \limsup _{r \rightarrow \infty} \frac{\bar{H}(r)}{\bar{F}(r)}<\infty .
$$

Proof. The structure of the argument is similar to that in Theorem 2.1. We also follow the conventions in the proof of Theorem 2.1 by working with the tail of the empty space function. However, since in this case the weight of a $\Phi$-ball is not a function of its radius, we have to view the Poisson random measure $\Phi$ as a measure in the full $d+2$-dimensional space $\mathbb{R}^{d} \times(0, \infty) \times(0, \infty)$.

As before, we start with proving the lower bound in (2.21). The scenario we will use is similar to the scenario we used to prove the lower bound in Theorem 2.1. Specifically, in this scenario there is a single ball of a large radius and large weight that "eliminates" all the other $\Phi$-balls that intersect $B_{r}(\mathbf{0})$, and then another ball in $\Phi$ of an even larger weight "eliminates" the first ball, but does not itself intersect $B_{r}(\mathbf{0})$. Recall the definition of the sets $A_{r}^{(1)}, A_{r}^{(2)}$ and $A_{r}^{(3)}$ in $(2.2)-(2.4)$ accordingly. As before, on the event

$$
B_{r}=\left\{\Phi\left(A_{r}^{(1)} \times(0, \infty)\right)=1\right\},
$$

we can define a random vector $\left(\mathbf{X}^{(r)}, R^{(r)}, W^{(r)}\right)$ corresponding to the location of the center, the radius and the weight of the single ball in $\Phi$ for which the pair $\left(\mathbf{X}^{(r)}, R^{(r)}\right)$ is in the set $A_{r}^{(1)}$. Therefore,

$$
P\left(B_{r}(\mathbf{0}) \cap D_{\mathrm{th}}=\emptyset\right) \geq P\left(B_{r} \cap \hat{B}_{r}\right),
$$

where now

$$
\begin{aligned}
\hat{B}_{r}=\{ & W^{(r)}>\max \left\{w:(\mathbf{x}, t, w) \in \Phi\left(\left(A_{r}^{(2)} \cup A_{r}^{(3)}\right) \times(0, \infty)\right)\right. \\
& \text { and there is }(\mathbf{x}, t, w) \in \Phi\left(\left(A_{r}^{(1)} \cup A_{r}^{(2)} \cup A_{r}^{(3)}\right)^{c}\right) \\
& \text { and } \left.w>W^{(r)} \text { such that } B_{t}(\mathbf{x}) \text { intersects } B_{R^{(r)}}\left(\mathbf{X}^{(r)}\right)\right\} .
\end{aligned}
$$

A standard computation shows that if (for example) $2 r \leq R^{(r)} \leq 3 r$, then the expected number of the Boolean balls that intersect $B_{R^{(r)}}\left(\mathbf{X}^{(r)}\right)$ but not $B_{r}(\mathbf{0})$ is at least $c r^{d}$. Similarly,

$$
P\left(\Phi\left(A_{r}^{(1)} \times(0, \infty)\right)=1,2 r \leq R^{(r)} \leq 3 r\right) \geq c r^{d} \bar{F}(r) .
$$

Since

$$
m\left(\left(A_{r}^{(2)} \cup A_{r}^{(3)}\right) \times(0, \infty)\right) \leq c r^{d},
$$

in order to prove the lower bound in (2.21) it is enough to prove the following statement. Let $c_{1}, c_{2}$ be positive numbers, and let $N_{1}, N_{2}$ be independent Poisson random variables with means $c_{1} r^{d}$ and $c_{2} r^{d}$, correspondingly. Let $W_{0}, W_{n}^{(1)}, n=1,2, \ldots, W_{n}^{(2)}, n=1,2, \ldots$ be i.i.d. standard uniform random variables independent of the Poisson random variables. Then for some positive $c$,

$$
P\left(\sup _{n \leq N_{2}} W_{n}^{(2)}>W_{0}>\sup _{n \leq N_{1}} W_{n}^{(1)}\right) \geq c r^{-d} .
$$


To this end, note that, by symmetry, for any fixed $n_{1} \geq 1, n_{2} \geq 1$,

$$
P\left(\sup _{n \leq n_{2}} W_{n}^{(2)}>W_{0}>\sup _{n \leq n_{1}} W_{n}^{(1)}\right)=\frac{n_{2}}{n_{1}+n_{2}+1} \cdot \frac{1}{n_{1}+1} .
$$

Now (2.22) follows from the fact that

$$
P\left(N_{2} \geq\left(c_{2} / 2\right) r^{d}\right) \rightarrow 1, \quad P\left(N_{1} \leq 2 c_{1} r^{d}\right) \rightarrow 1
$$

as $r \rightarrow \infty$. This completes the proof of the lower bound.

Now we prove the upper bound in (2.21). Let $K_{1}$ be a large positive number we will specify below. Denote

$A_{r}^{(7)}\left(K_{1}\right)=\left\{(\mathbf{x}, s, w) \in \mathbb{R}^{d} \times(0, \infty) \times(0, \infty): s \geq r / K_{1}\right.$, the ball $B_{s}(\mathbf{x})$ intersects the ball $\left.B_{r}(\mathbf{0})\right\}$. As in (2.9) we have

$$
E\left[\Phi\left(A_{r}^{(7)}\left(K_{1}\right)\right)\right] \leq c r^{d} \bar{F}(r)
$$

(with a $K_{1}$-dependent constant $c$ ). Therefore, if $l_{1} \geq \alpha /(\alpha-d)$, then

$$
P\left(\Phi\left(A_{r}^{(7)}\left(K_{1}\right)\right)>l_{1}\right)=o(\bar{F}(r))
$$

as $r \rightarrow \infty$, and, hence, we need to prove that

$$
\limsup _{r \rightarrow \infty} \frac{P\left(B_{r}(\mathbf{0}) \cap D_{\mathrm{th}}=\emptyset, \Phi\left(A_{r}^{(7)}\left(K_{1}\right)\right) \leq l_{1}\right)}{\bar{F}(r)}<\infty .
$$

Fix $\theta \in(d / \alpha, 1)$, and let

$$
A_{r}^{(8)}\left(K_{1}, \theta\right)=\left\{(\mathbf{x}, s, w) \in \mathbb{R}^{d} \times(0, \infty) \times(0, \infty): r^{\theta}<s<r / K_{1},\right.
$$

the ball $B_{s}(\mathbf{x})$ intersects the ball $\left.B_{r}(\mathbf{0})\right\}$.

As above, we have for large $r$,

$$
E\left[\Phi\left(A_{r}^{(8)}\left(K_{1}, \theta\right)\right)\right] \leq c r^{d} \bar{F}\left(r^{\theta}\right) .
$$

By the choice of $\theta$ we see that, if $l_{2} \geq \alpha /(\theta \alpha-d)$, then

$$
P\left(\Phi\left(A_{r}^{(8)}\left(K_{1}, \theta\right)\right)>l_{2}\right)=o(\bar{F}(r))
$$

as $r \rightarrow \infty$. Therefore, in order to establish (2.25), it is enough to prove that for every $j=0,1, \ldots, l_{1}$,

$$
\limsup _{r \rightarrow \infty} \frac{P\left(B_{r}(\mathbf{0}) \cap D_{\mathrm{th}}=\emptyset, \Phi\left(A_{r}^{(7)}\left(K_{1}\right)\right)=j, \Phi\left(A_{r}^{(8)}\left(K_{1}, \theta\right)\right) \leq l_{2}\right)}{\bar{F}(r)}<\infty .
$$

Now we specify $K_{1}$ by setting $K_{1}>l_{2}$. Note that for every choice of $l_{2}$ and $K_{1}$ as above, the complement in $B_{r}(\mathbf{0})$ of the union of at most $l_{2}$ balls of radii not exceeding $r / K_{1}$ contains a ball of a radius $\gamma r$ for some $\gamma=\gamma\left(l_{2}, K_{1}\right)>0$ (we can choose $\gamma=\left(1 / l_{2}-1 / K_{1}\right) / 2$ ).

We first consider the case $j=0$ in (2.26). Let $\Phi_{r, \theta}$ be the restriction of the Poisson random measure $\Phi$ to the set $\mathbb{R}^{d} \times\left(0, r^{\theta}\right] \times(0, \infty)$. Using, once again, the property of a Poisson measure that its restrictions to disjoint sets are independent, we see that $(2.26)$ with $j=0$ will follow once we show that

$$
\limsup _{r \rightarrow \infty} \frac{P\left(B_{\gamma r}(\mathbf{0}) \cap D_{r, \theta, \text { th }}=\emptyset\right)}{\bar{F}(r)}<\infty,
$$

where $D_{r, \theta \text {,th }}$ is the grain cover corresponding to the thinning of $\Phi_{r, \theta}$ (with the weights still being independent of the radii). The fact that we are allowed to use the ball centered at the origin in 
(2.27), instead of a randomly centered ball described in the previous paragraph, is a consequence of translation invariance of $\Phi_{r, \theta}$.

In order to prove (2.27), we need one more simple estimate. Let $t$ be a large number, $t \leq \gamma r / 3$. Consider concentric balls $B_{t / 3}(\mathbf{0}), B_{t}(\mathbf{0})$ and $B_{3 t}(\mathbf{0})$. Then there is $0<q<1$ such that

$$
\begin{aligned}
P(\sup \{w:(\mathbf{x}, s, w) & \left.\in \Phi_{r, \theta}\left(\left(B_{t}(\mathbf{0}) \backslash B_{t / 3}(\mathbf{0})\right) \times(0, \infty)\right)\right\} \\
>\max (\sup \{w:(\mathbf{x}, s, w) & \left.\in \Phi_{r, \theta}\left(\left(B_{t / 3}(\mathbf{0}) \backslash B_{t / 9}(\mathbf{0})\right) \times(0, \infty)\right)\right\}, \\
\sup \{w:(\mathbf{x}, s, w) & \left.\left.\in \Phi_{r, \theta}\left(\left(B_{3 t}(\mathbf{0}) \backslash B_{t}(\mathbf{0})\right) \times(0, \infty)\right)\right\}\right) \geq q
\end{aligned}
$$

for all $t$ large enough. Indeed, the Poisson random measure $\Phi_{r, \theta}$ assigns mean measures of the order $c t^{d}$ to each of the three annuli in question (with $c K_{1}$-dependent), so (2.28) follows by using conditioning and a computation analogous to (2.23).

Now it is clear that the probability in the numerator in (2.27) can be bounded from above by $(1-q)^{c r^{1-\theta}}$ for some $c>0$ because we can fit into $B_{\gamma r}(\mathbf{0})$ triple annuli as above with the radial separation between neighboring triples exceeding $r^{\theta}$, which makes, by the definition of $\Phi_{r, \theta}$, the events whose probabilities are computed in (2.28), independent. Therefore, (2.27) holds, and so we have proved (2.26) with $j=0$.

Next we consider (2.26) with $j=1$. It follows from (2.24) that

$$
P\left(\Phi\left(A_{r}^{(7)}\left(K_{1}\right)\right)=1\right)=O\left(r^{d} \bar{F}(r)\right)
$$

as $r \rightarrow \infty$. Therefore, we need to prove the following version of (2.27): consider the grain cover $D_{r, \theta, \text { th }}$ and a random variable $W$ independent of it, whose law is the distribution of the weight in the Boolean model ( $W$ is the weight of the single ball in $\Phi\left(A_{r}^{(7)}\left(K_{1}\right)\right)$. We eliminate all the balls in $D_{r, \theta, \text { th }}$ whose weight is smaller or equal to $W$, and we call the resulting grain cover $\hat{D}_{r, \theta, \mathrm{th}}$. Then (2.26) with $j=1$ will follow once we prove that

$$
\limsup _{r \rightarrow \infty} r^{d} P\left(B_{\gamma r}(\mathbf{0}) \cap \hat{D}_{r, \theta, \text { th }}=\emptyset\right)<\infty .
$$

In order to see that this is true, we use an argument similar to the one used to prove (2.27). Consider the triple annuli in (2.28). Since we already know that the probability that fewer than $c r^{1-\theta}$ events in (2.28) occurs is $o\left(r^{-d}\right)$, we only need to consider what happens if at least $c r^{1-\theta}$ of

the events occur. In the latter case, the only possibility for $B_{\gamma r}(\mathbf{0}) \cap \hat{D}_{r, \theta, \text { th }}=\emptyset$ is that the weight of the heaviest $\Phi$-ball in the union of $c r^{1-\theta}$ of annuli of radii of order $c r$ and width of order $c r^{\theta}$ does not exceed $W$. Since the mean measure of the Poisson random measure $\Phi_{r, \theta}$ assigns the weight of the order $\mathrm{cr}^{d}$ to that union, the latter probability does not exceed $c r^{-d}$, once again, by conditioning and a computation analogous to $(2.23)$.

Therefore, (2.29) is true, and so we have proved (2.26) with $j=1$. The cases $j=2, \ldots, l_{1}$, are similar and easier, since the probabilities

$$
P\left(\Phi\left(A_{r}^{(7)}\left(K_{1}\right)\right)=j\right)
$$

become asymptotically smaller as $j$ increases.

This completes the proof of the upper bound in (2.21).

\section{Heavy small balls and exponential decay of the CONTACT Distribution}

In this section we prove that, if the weight of a ball is a strictly decreasing function of its radius, then the tail of the contact distribution decays exponentially fast. This turns out to be unrelated to the fact that the tail of the radii of the balls is regularly varying. 
Theorem 3.1. Assume that the distribution of the radii of the balls in the Boolean model satisfies (1.2). If larger balls have smaller weights, then for some $c>0$ the contact distribution of the thinned germ-grain model satisfies

$$
\bar{H}(r) \leq e^{-c r^{d}}
$$

for all $r$ large enough.

Proof. Once again, we work with the tail of the empty space function. Since the weight of a ball is a function of its radius, we switch, once again, to viewing the Poisson random measure $\Phi$ as a measure in the $d+1$-dimensional space $\mathbb{R}^{d} \times(0, \infty)$.

Choose a finite number $\gamma>0$ such that

$$
F((0, \gamma))>0 .
$$

Let $\Phi_{\gamma}$ be the restriction of the Poisson random measure $\Phi$ to the set $\{(\mathbf{x}, s): s \leq \gamma\}$. As in the proof of Theorem 2.2, for some $c>0$ that depends on $\gamma$, we can find at least $c r^{d}$ disjoint balls of radius $\gamma$ within $B_{r}(\mathbf{0})$, such that the distance between any two different balls exceeds $2 \gamma$. Let us call these balls $B_{i}, i=1, \ldots, n$, with $n \geq c r^{d}$. For $i=1, \ldots, n$ consider the event

$H_{i}=\left\{\Phi_{\gamma}\left(\left\{(\mathbf{x}, s): B_{s}(\mathbf{x}) \cap B_{i} \neq \emptyset\right\}\right)=1, \Phi_{\gamma}\left(\left\{(\mathbf{x}, s): B_{s}(\mathbf{x}) \cap\left[\left(B_{i}+B_{\gamma}(\mathbf{0})\right) \backslash B_{i}\right] \neq \emptyset\right\}\right)=0\right\}$.

Here $B_{i}+B_{\gamma}(\mathbf{0})$ is simply the ball concentric with $B_{i}$ of radius $2 \gamma$. Note that, on the event $H_{i}$, the single $\Phi_{\gamma}$-ball in the description on the event cannot be "eliminated" by any other $\Phi$-ball. Indeed, if another $\Phi_{\gamma}$-ball intersected it, the latter ball would be in the set $\left[\left(B_{i}+B_{\gamma}(\mathbf{0})\right) \backslash B_{i}\right]$, which is impossible on the event $H_{i}$. Furthermore, any $\Phi$-ball which is not a $\Phi_{\gamma}$-ball has simply too large a radius. Therefore,

$$
\begin{aligned}
& P\left(B_{r}(\mathbf{0}) \cap D_{\mathrm{th}}=\emptyset\right) \leq P\left(\cap_{i=1}^{n} H_{i}^{c}\right) \\
= & \left(1-P\left(H_{1}\right)\right)^{n} \leq\left(1-P\left(H_{1}\right)\right)^{c r^{d}} .
\end{aligned}
$$

The equality in this calculation follows from the fact that the balls $B_{i}$ are sufficiently far away from each other so that different events $H_{i}$ are determined by restrictions of the Poisson random measure $\Phi_{\gamma}$ to disjoint sets and, hence, are independent.

In order to prove the theorem we only need to check that $P\left(H_{1}\right)>0$. Since $\Phi_{\gamma}$ is translation invariant, we replace, in the calculation below, $B_{1}$ by $B_{\gamma}(\mathbf{0})$ and $B_{1}+B_{\gamma}(\mathbf{0})$ by $B_{2 \gamma}(\mathbf{0})$. Note that the event $H_{1}$ is defined as the intersection of two independent events, so we only need to check that each one of these events has a positive probability.

It is clear that

$$
\Phi_{\gamma}\left(B_{\gamma}(\mathbf{0}) \times(0, \gamma]\right) \subset \Phi_{\gamma}\left(\left\{(\mathbf{x}, s): B_{s}(\mathbf{x}) \cap B_{\gamma}(\mathbf{0}) \neq \emptyset\right\}\right) \subset \Phi_{\gamma}\left(B_{2 \gamma}(\mathbf{0}) \times(0, \gamma]\right),
$$

so that

$$
E\left[\Phi_{\gamma}\left(\left\{(\mathbf{x}, s): B_{s}(\mathbf{x}) \cap B_{\gamma}(\mathbf{0}) \neq \emptyset\right\}\right)\right] \in(0, \infty)
$$

and, hence,

$$
P\left(\Phi_{\gamma}\left(\left\{(\mathbf{x}, s): B_{s}(\mathbf{x}) \cap B_{\gamma}(\mathbf{0}) \neq \emptyset\right\}\right)=1\right)>0 .
$$

Further,

$$
\left.\Phi_{\gamma}\left(\left\{(\mathbf{x}, s): B_{s}(\mathbf{x}) \cap\left(B_{2 \gamma}(\mathbf{0})\right) \backslash B_{\gamma}(\mathbf{0})\right) \neq \emptyset\right\}\right) \subset \Phi_{\gamma}\left(B_{3 \gamma}(\mathbf{0}) \times(0, \gamma]\right),
$$

so that

$$
E\left[\Phi_{\gamma}\left(\left\{(\mathbf{x}, s): B_{s}(\mathbf{x}) \cap\left(B_{2 \gamma}(\mathbf{0})\right) \backslash B_{\gamma}(\mathbf{0})\right) \neq \emptyset\right\}\right]<\infty
$$


and, hence,

$$
P\left(\Phi_{\gamma}\left(\left\{(\mathbf{x}, s): B_{s}(\mathbf{x}) \cap\left(B_{2 \gamma}(\mathbf{0})\right) \backslash B_{\gamma}(\mathbf{0})\right) \neq \emptyset\right\}=0\right)>0 .
$$

This implies that $P\left(H_{1}\right)>0$, and the proof of the theorem is complete.

Notice that a lower bound of the type

$$
\bar{H}(r) \geq e^{-c r^{d}}
$$

for large $r$ (with, possibly, a different exponent $c$ than in (3.1)) is trivially true since it holds for the original spherical Boolean model even before thinning.

\section{REFERENCES}

J. Anderson, O. HäGgström and M. MAnsson (2006): The volume fraction of a nonoverlapping germ-grain model. Electronic Communications in Probability 11:78-99.

D. Hug, G. Last and W. Weil (2002): A survey on contact distribution. In Morphology of Condensed Matter, K. Mecke and D. Stoyan, editors, volume 600 of Lecture Notes in Physics. Springer-Verlag, Berlin, pp. 317-357.

M. KuRONEN and L. LESKELÄ (2013): Hard-core thinning of germ-grain models with power-law grain sizes. Advances in Applied Probability 45:595-625.

M. Mansson and M. Rudemo (2002): Random patterns of nonoverlapping convex grains. $A d-$ vances in Applied Probability 34:718-738.

T. NGuYen and F. BACCELLI (2013): On the generating functionals of a class of random packing point processes. Preprint.

S. Resnick (2007): Heavy-Tail Phenomena: Probabilistic and Statistical Modeling. Springer, New York.

D. Stoyan, W. Kendall and J. Mecke (1995): Stochastic Geometry and its Applications. Wiley.

College of Mathematics and Statistics, Nanjing University of Information Science and TechnolOGY, NANJING 210044, CHINA

E-mail address: dongyinghua1@163.com

School of Operations Research and Information Engineering, and Department of Statistical Science, Cornell University, IthacA, NY 14853

E-mail address: gs18@cornell.edu 